\begin{tabular}{l}
\hline Alimentary Pharmacology \\
\hline \& Therapeutics \\
\hline
\end{tabular}

\title{
Original article: Natural history of acute colonic diverticular bleeding: prospective study in 133 consecutive patients
}

\begin{tabular}{|r|l|}
\hline Journal: & Alimentary Pharmacology \& Therapeutics \\
\hline Manuscript ID: & APT-0162-2010.R1 \\
\hline Manuscript Type: & Original Scientific Paper \\
\hline Author: & 11 -May-2010 \\
\hline Komplete List of Authors: & $\begin{array}{l}\text { Poncet, Gilles; University Hospital, Alimentary Tract Surgery } \\
\text { Heluwaert, Frederic; General Hospital, Gastroenterology } \\
\text { Voirin, David; University Hospital, Alimentary Tract Surgery } \\
\text { Bonaz, Bruno; University Hospital, Gastroenterology } \\
\text { Faucheron, Jean-Luc; University Hospital, Alimentary Tract Surgery }\end{array}$ \\
\hline Keywords: & $\begin{array}{l}\text { Enteric infections < Disease-based, Large intestine < Organ-based, } \\
\text { Surgery < Topics, Outcomes research < Topics, Colonoscopy < } \\
\text { Topics }\end{array}$ \\
\hline
\end{tabular}

\section{(5) ScholarONE


Original article: Natural history of acute colonic diverticular bleeding: prospective study in 133 consecutive patients

Short running title: Colonic diverticular bleeding

Authors: Gilles Poncet ${ }^{*}, \mathrm{MD}, \mathrm{PhD}$, Frederic Heluwaert ${ }^{\dagger}, \mathrm{MD}$, David Voirin ${ }^{*}, \mathrm{MD}$, Bruno Bonaz $^{\ddagger}, \mathrm{MD}, \mathrm{PhD}$, Jean-Luc Faucheron ${ }^{*}, \mathrm{MD}, \mathrm{PhD}$.

\section{Institutions:}

${ }^{1}$ Colorectal Unit, Department of Surgery, Grenoble University Hospital, Grenoble, France

†Department of Gastroenterology, Annecy General Hospital, Annecy, France

${ }^{\ddagger}$ Department of Gastroenterology, Grenoble University Hospital, Grenoble, France

Correspondence to: Prof. Jean-Luc Faucheron, Colorectal Unit, Department of Surgery, Michallon University Hospital, B.P. 217, 38043 Grenoble cedex, France

Tel: 33.4.76.76.53.71. Fax: 33.4.76.76.87.80. E-mail: JLFaucheron@ @hu-grenoble.fr

Source of support and Disclosure: none

Acknowledgements: the authors thank Ms Francesca Corrado for her English writing support

Keywords: Diverticular disease; Lower gastrointestinal haemorrhage; Therapeutic strategy; Endoscopy; Surgery 


\section{SUMMARY}

Ba und: Bleeding recurrence rate after spontaneous haemostasis of colonic diverticular ha€ $\equiv$ Iage varies in the literature, and a small minority of patients will require endoscopic, radiological or surgical intervention.

Aim: To study the natural history of colonic diverticular bleeding in consecutive patients.

Methods: We prospectively studied consecutive patients admitted for colonic diverticular bleeding from 1997 to 2005. Data on age, gender, 30-day mortality, therapeutic modality for bleeding management, and subsequent rebleeding were collected.

Results: One hundred and thirty three patients (mean age 75.7 years) were recruited. Bleeding stopped spontaneously in 123 patients $(92.4 \%)$. A more interventional approach was necessary in 10 patients. Thirty-day mortality rate for first bleeding was $2.25 \%$. Out of the 123 patients managed conservatively and submitted to an average follow-up of 47.5 months, $17(13.8 \%)$ presented at least one recurrent diverticular bleeding. Spontaneous haemostasis was obtained in all recurrent cases except one, who died. The estimated bleeding recurrence rate was $3.8 \%$ at one year, $6.9 \%$ at 5 years and $9.8 \%$ at 10 years.

Conclusions: The low estimated rebleeding rate and the fact that rebleeding can be treated conservatively in most cases suggest that aggressive approach is not justified.

Comment [UW2]: Specific

Comment [UW3]: Background and aim of the study have been slightly changed, as we agree with the remarks from reviewers $2 \& 3$ : the work is aimed at studying the natural history of acute diverticular bleeding rather than to support or refute colectomy as a primary treatment 


\section{INTRODUCTION}

Colonic diverticular disease is common in Western countries, with a prevalence increasing from less than $10 \%$ in adults under 40 to about $60 \%$ in those over $80 .{ }^{1}$ The disease remains asymptomatic in $80 \%$ of affected individuals, while the rest develops complications. The most frequent complication is diverticulitis with the risks of abscess formation, fistulation, perforation and obstruction. ${ }^{2}$ The second complication is acute diverticular bleeding, affecting $3 \%$ to $15 \%$ of individuals with diverticular disease. ${ }^{3-6}$ Diverticular haemorrhage usually stops spontaneously in 70 to $90 \%$ of cases. ${ }^{7-9}$ The rate of rebleeding after one episode is between 20 to $38 \%$, hence aggressive search for bleeding site and elective colectomy being sometimes proposed. ${ }^{8,10-12}$ This study was performed to provide the latest and most accurate answers to the following questions: $i$ ) what proportion of bleeding in patients with colonic diverticular stop spontaneously, ii) what is the 30-day mortality rate after the first episode of bleeding, iii) what proportion of patients bleed again and what is the outcome?

\section{PATIENTS AND METHODS}

\section{Patients}

We prospectively recruited consecutive patients who came to our institution with colonic diverticular bleeding from January 1997 to August 2005, among 1145 patients admitted for acute lower gastrointestinal (GI) bleeding in our GI bleeding intensive care unit and

Comment [UW4]: This is the only point from the reviewers comments we did not change, as the study is a true prospective one (first minor comment from reviewer 3 ). We develop our arguments in the separate sheet and slightly changed the text in that section, trying to be more convincing.. prospectively evaluated. All these patients had a systematic colonoscopy on entry as part of a pro $\equiv$ in our intensive care unit for lower GI bleeding. Diagnosis of diverticulosis as the source of haemorrhage was established according to the criteria proposed by Zuckerman ${ }^{13}$ and Jensen. ${ }^{3}$ These included evident endoscopic stigmata such as active bleeding, visible vessel and adherent clot, and presumptive findings such as presence of fresh blood within one or more colonic segments and diverticular erosions. Evidence of diverticular bleeding was also 
confirmed in some cases and for therapeutic objectives by helical CT angiography $(n=4)$ or selective mesenteric arteriography $(\mathrm{n}=7)$ showing vascular extravasation within the colonic segment with diverticulosis at endoscopic examination.

\section{Standardized protocol}

We cted data on age, gender, symptoms on inclusion, anti-inflammatory drug intake [non steroraa anti-inflammatory drugs (NSAID) and steroids], anti-coagulants and anti-platelets medication (including aspirin) and delay between the first intake of these medications and the bleeding episode. We also recorded initial haemoglobin level measured on admi $\equiv$ haemoglobin level observed during hospitalization, discharge haemoglobin level, admission platelet level, admission prothrombin level, number of packed red cell units transfused, length of hospital stay, and 30-day mortality. Therapeutic modality for the bleeding management was reported. In our GI bleeding intensive care unit standardized protocol, anti-coagulants were systematically stopped, antagonized by prothrombin complex, and replaced by heparin; NSAID were systematically discontinued; anti-platelets were stopped each time it was possible, depending on the patient's cardiac condition (on systematic cardiologist advice requested in emergency) and bleeding severity.

Prospective evaluation including data on subsequent bleeding was obtained by means of patients' examination including interview at regular intervals.

\section{Statistics}

Data were expressed as mean (range). Chi-square vas used to compare data. A p value of less than 0.05 was considered as significant. Recurrence rate was calculated using the KaplanMeier method.

\section{RESULTS}

Patients' demographics
Comment [UW5]: This paragraph hs been created to answer remarks from reviewers 1 \& 3

Comment [UW6]: VKA was replaced by anti-coagulant throughout the manuscript, to be more uniform and as it is the more usual term (answer to reviewer 1)

Comment [UW7]: The brand name 'Kaskadil' has been deleted as requested by reviewer 3 in his minor comments. This is also an answer to the fourth comment from reviewer 1

Comment [UW8]: This is the policy we use in the protocol that is ongoing in the GI bleeding intensive care unit (answer to reviewers 1 
1

3

4

5

6

7

8

9

10
During the study period, 133 consecutive patients $(73$ men) with a mean age of 75.7 years (range 48-97) presenting with 157 diverticular bleeding episodes, were recruited. Women were significantly younger than men $(72.3$ vs. 79.9 years, $\mathrm{p}<0.05)$. Thirty four patients (25.6\%) presented with a systolic blood pressure below $80 \mathrm{~mm} \mathrm{Hg}$ on admission, seven of whom had lost consciousness. No patient had abdominal pain or fever. Biological data are shown in table 1.

\section{Immediate outcome}

A total of 74 patients $(55.6 \%)$ required blood transfusion, with a mean of 3.8 units of packed red 1 cells units per transfused patient (range 1-14) because of severe anaemia. Sixty two patients received blood transfusion during the first episode and 12 during the second episode of bleeding. The average length of stay in the GI bleeding unit was 9.2 days (range 3-38). Although bleeding had spontaneously stopped, 3 patients (aged 71, 88, and 89 years) died during the first 30 days (mortality rate of $2.25 \%$ for the first bleeding) from multiple organ failure in the first patient, stroke in the second and both terminal respiratory and neurologic diseases in the third patient.

Out of the 133 patients, the first bleeding stopped spontaneously in $123(92.4 \%)$.

In ten patients, a more aggressive approach was mandatory because of the severity of the bleeding with no spontaneous haemostasis. The criteria for this aggressive approach were a persistent bleeding with hemodynamic instability despite transfusion of 3 or more packed red cells units, interruption of medication if present and if possible in terms of cardiovascular indication, treatment with prothrombin complex and/or fresh frozen plasma if needed, and standard resuscitation manoeuvres. Three patients were treated endoscopically (epinephrine injection in 3, clipping in 2), and was operated in an emergency (Hartmann's procedure) because of failure to obtain haemostasis by injection of diluted epinephrine and clipping. Four other patients were treated by arteriography followed by immediate superselective
Comment [UW10]: See UW 8

Comment [UW11]: Answer to comment from reviewer 2 
embolization leading to a secondary Hartmann's procedure for ischemic lesions in one. Lastly, 3 other patients required emergency surgery: the site of diverticular bleeding was confirmed by angiography in 1 and helical CT scan in 1, and both patients underwent left colectomy with primary anastomosis; the last one was treated by a non restorative subtotal colectomy for non-identified site of the rebleeding diverticula.

\section{Further follow-up}

Excluding the 5 patients who had surgery during the first hospital stay, the 3 patients who die $\equiv 2$ patients who were lost to follow up, 123 patients were submitted to a median follow-up of 47.5 months (range 5-136). Interestingly, one patient who required an emergency left colectomy was readmitted for a rebleeding diverticulosis 14 months later and was followed by simple surveillance.

Among the 123 patients who were managed conservatively, 17 (13.8\%) presented at least one recurrent diverticular bleeding within 38 months in average (range 2-136).

Among the 12 patients with only one rebleeding, 3 received blood transfusion of respectively 2, 4, and 6 units of packed red blood cells. One patient had a successful endoscopic haemostasis (clip). The other 11 patients were followed conservatively and had spontaneous haemostasis. No risk factor for rebleeding was identified in this series (Table 2).

Four patients had two diverticular bleeding recurrences during the follow-up and two patients among them received respectively 2 and 3 units of red blood cells for the first recurrent episode and 3 patients received respectively 3,3 and 5 units of red blood cells for the second bleeding episode. A spontaneous haemostasis was obtained in all cases. An 87-year old patient from that group died from multiple organ failure during hospitalization for the second bleeding recurrence. 
1

3

4

5

6

7

8

9

10

One patient presented with 3 diverticular rebleeding episodes required a blood transfusion (10 units) during the first one and none for the following. Each time, haemostasis occurred spontaneously.

The estimated bleeding recurrence rate from colonic diverticula (Kaplan Meier method) was $3.8 \%$ at one year, $6.9 \%$ at 5 years and $9.8 \%$ at 10 years in this series. One patient among the $17(5.8 \%)$ presenting with a colonic diverticular rebleeding died.

\section{DISCUSSION}

Diverticular disease of the colon is the fifth most important gastrointestinal disease in terms of direct and indirect health care costs in western countries. ${ }^{14}$ The colonic origin of lower GI haemorrhage, in order of decreasing incidence, is diverticulosis, inflammatory bowel disease, colonic neoplasia, infectious and ischemic colitis, benign anorectal disease, and arteriovenous malformations. ${ }^{15}$ Positive diagnosis of bleeding diverticulosis has evolved during the last decade with the development of new technologies such as CT helical angiography and selective mesenteric angiography when the colonoscopy is not feasible, contra-indicated, incomplete or non contributive. ${ }^{15,16}$ Management has also evolved with the advent of interventional endoscopy (bipolar coagulation, epinephrine injection, clip placement) ${ }^{11,15,17,18}$ and angiographic treatment (intra-arterial infusion of vasopressin, transarterial embolization). ${ }^{19-22}$ In the 10 patients treated on this last series and whose massive diverticular bleeding did not stop spontaneously, we could foresee haemostasis endoscopically in 2 and superselective embolization in 4 .

Diverticular bleeding is caused by the rupture of small arteries, explaining the frequency of sudden haemorrhage associated with low blood pressure at admission, as observed in more than $25 \%$ of patients in this series. NSAIDs and high dose aspirin are responsible for early bleeding (one week after first medication intake). ${ }^{23}$ Anti-coagulants, low dose aspirin, 
clopidogrel and steroids seem to be associated with late bleeding (one year after first medication intake).

Diverticular bleeding usually stops spontaneously in 70 to $90 \%$ of cases. ${ }^{7-9,15}$ In the present series, bleeding ceased spontaneously in $92.4 \%$ of cases, which is slightly higher than the previously published rates. The reason for this is probably due to multiple factors; the first one might be the highly specialization of the GI bleeding intensive care unit in which the team was used to manage such conditions, avoiding unnecessary, too early therapeutic endoscopy, arteriography or surgery; the second reason is that anti-coagulants, anti-platelets or NSAID medications were discontinued in the majority of cases whenever possible; the third reason is that gastroenterologists and surgeons from the same team carefully followed patients together, in order not to miss the time of surgery, when needed.

Thirty day mortality rate in this series is $2.25 \%$ ( 3 patients out of 133 ) for the first diverticular bleeding and 5.8\% (1 patient out of 17) for the subsequent bleeding episodes. In all of these cases, haemostasis was spontaneously obtained by medical management and deaths occurred because of severe underlying conditions, in patients older than the others in the same series. Mortality rates in the literature vary from $0{ }^{3}$ to $40 \% .{ }^{24}$ These wide variations are due to many reasons, such as time of publication, method of treatment used, association with other conditions, severity of the disease, or selection of patients. In a recent national population study, 30-day mortality following diverticular disease hospital admission was $5.1 \%$ and 1 year mortality was $14.5 \%{ }^{25}$ Increasing age, comorbidity and emergency admission as for diverticular bleeding were independent predictors of mortality. ${ }^{25}$ For instance, it has been proven that mortality increases significantly when patients receive more than 10 units of blood, ${ }^{22}$ or when patients require emergency total colectomy for non localized colonic diverticular bleeding by a non specialized surgeon. In a collected series of patients with surgery for bleeding diverticulosis, reviewed by Bender et al., ${ }^{26}$ there was a cumulative 
mortality rate of $22.6 \%$ in all series from 1972 to 1990 . Interestingly, the mean age of the patients in our series (75.7 years) is far higher than those usually published in the literature, so that a mortality rate of $2.25 \%$ might seem not too high.

The rebleeding rate in this series is $13.8 \%$ (17 patients out of 123 stopped bleeding after conservative management) within a mean time of 47.5 months. This rate compares favourably with most of the rates published in the literature. In 1994, McGuire stated that "the rebleeding rate after one episode was usually said to be $20 \%$, a rate that might have been diluted by inclusion of other diagnoses" ${ }^{8}$ Twenty eight of the 73 patients (38.4\%) from his series rebled at a mean follow-up of 4.3 years, 6 of whom required an emergency surgery and 22 stopped spontaneously. ${ }^{8}$ Longstreth ${ }^{10}$ and later on Hoedema et al. ${ }^{22}$ reported rebleeding rates of 25 to $30 \%$. The difference between these studies and our work might be the length of follow-up, which is a little shorter in our series (47.5 months). Another explanation might be the patients lost on follow-up; if we assume that the 2 patients who never came to their follow-up in our series might have rebled, the percentage would have been $15.2 \%$ (19 patients out of 125), which is closer to the published rates of 20 to $38 \%$. But the most obvious cause is probably the aggressive approach we had erning the first bleeding: endoscopic haemostasis in 3 patients, embolization in 4 , and medication discontinuation in the majority of patients being on anti-coagulants, anti-platelets or NSAIDs; Jensen et al. reported a bleeding recurrence rate of $0 \%$ when the 10 patients of their series underwent endoscopic treatment (epinephrine injection, bipolar coagulation, or both), instead of a recurrence rate of $52.9 \%$ in the 17 patients who only had simple surveillance after colonoscopy. ${ }^{3}$ The authors concluded that colonoscopic treatment of such patients may prevent recurrent bleeding and decrease the need for surgery. In the study of Bloomfeld, although the haemostatic procedures were similar to the previous study, results were less encouraging: among 13 patients with active diverticular bleeding endoscopically managed, 5 and 3 respectively presented an early or a late recurrence 
up to 35 months, thus corresponding to a success rate of $38.4 \% .^{11}$ Smoot et al. reported neither recurrence nor complication following endoscopic haemostasis carried out in 7 patients actively bleeding from colonic diverticula (follow-up period not stated). ${ }^{27}$ Finally, with the more recent review of Pilichos et al. presenting the results of all relevant studies and reports on this topic, one could write that out of 63 patients endoscopically treated, $6(9.5 \%)$ presented an early relapse of bleeding and non method-related complication, although the follow-up period was only mentioned for 17 patients. ${ }^{17}$

Once bleeding has recurred and ceased spontaneously, the rebleeding rate increases and is close to $50 \%$, as reported by Breen et al. ${ }^{28}$ We observed a slightly lower rate of $29.4 \%$, as 5 patients out of 17 presented with more than one rebleeding. We also noticed that further bleeding recurrences were not especially more severe than the first recurrence, and that spontaneous haemostasis could be obtained in all cases.

The estimated bleeding recurrence rate from colonic diverticula in our series was 3.8\% at one year, $6.9 \%$ at 5 years and $9.8 \%$ at 10 years, which is far lower than the previous rate published in the literature. ${ }^{8,10}$ An 87 -year old patient died early from multiple organ failure after a second recurrence, giving a mortality rate for rebleeding of 5.8\%. These data argue that there is no place for a preventive colonic resection after a spontaneous haemostasis of a colonic diverticula bleeding. Moreover, one of our patients operated in emergency for persisting haemorrhage later presented a recurrent diverticular bleeding.

\section{Conclusion}

In this prospective study, the first colonic diverticular bleeding stopped spontaneously in 92.4\% of cases. In the remaining patients, haemostasis was obtained using endoscopic treatment, embolization, and/or surgery. Among the 123 patients with a mean follow-up of 47.5 months, $13.8 \%$ presented with at least one bleeding recurrence, none of whom required 


\begin{abstract}
surgery. The low estimated rebleeding rate of $9.8 \%$ at ten years, associated with the fact that rebleeding could be managed conservatively in this series argue for a non aggressive approach to prevent recurrence.
\end{abstract}




\section{References}

1. Munson KD, Hensien MA, Jacob LN et al. Diverticulitis. A comprehensive follow-up. Dis Colon Rectum 1996; 39: 318-22.

2. Parks TG. Natural history of diverticular disease of the colon. J Clin Gastroenterol 1975; 4: 53-69.

3. Jensen DM, Machicado GA, Jutabha R et al. Urgent colonoscopy for the diagnosis and treatment of severe diverticular hemorrhage. N Eng J Med 2000; 342: 78-82.

4. Jacobs DO. Clinical practice. Diverticulitis. N Eng J Med 2007; 357: 2057-66.

5. Szojda MM, Cuesta MA, Mulder CM, Felt-Bersma RJ. Review article: management of diverticulitis. Aliment Pharmacol Ther 2007; 26 Suppl 2: 67-76.

6. Tursi A, Papagrigoriadis S. Review article: the current and evolving treatment of colonic diverticular disease. Aliment Pharmacol Ther 2009; 30: 532-46.

7. Goldberg SM, Nivatvongs S, Rothenberger DA. Diverticular disease with acute hemorrhage. In: Schwartz SI, Shires GT, Spencer FC, ed. Principles of Surgery. McGrawHill: New-York, 1989.

8. McGuire HH Jr. Bleeding colonic diverticular. A reappraisal of natural history and management. Ann Surg 1994; 220: 653-6.

9. Myron L. Bleeding colonic diverticular. J Clin Gastroenterol 2008; 42: 1156-8.

10. Longstreth GF. Epidemiology and outcome of patients hospitalized with acute lower gastrointestinal hemorrhage: a population-based study. Am J Gastroenterol 1997; 92: 419-24.

11. Bloomfeld RS, Rockey DC, Shetzline MA. Endoscopic therapy of acute diverticular hemorrhage. Am J Gastroenterol 2001; 96: 2367-72.

12. Czymek R, Kempf A, Roblick U et al. Factors predicting the postoperative outcome of lower gastrointestinal hemorrhage. Int J Colorectal Dis 2009; 24: 983-8. 
13. Zuckerman GR, Prakash C. Acute lower intestinal bleeding: part I: clinical presentation and diagnosis. Gastrointest Endosc 1998; 48: 606-17.

14. Petruzziello L, Iacopini F, Bulajic M, Shah S, Costamagna G. Review article: uncomplicated diverticular disease of the colon. Aliment Pharmacol Ther 2006; 23: 1379-91. 15. Vernava AM $3^{\text {rd }}$, Moore BA, Longo WE et al. Lower gastrointestinal bleeding. Dis Colon Rectum 1997; 40: 846-58.

16. Ernst O, Bulois P, Saint-Drenant S et al. Helical CT in acute lower gastrointestinal bleeding. Eur Radiol 2003; 13: 114-7.

17. Pilichos C, Bobotis E. Role of endoscopy in the management of acute diverticular bleeding. World J Gastroenterol 2008; 14: 1981-3.

18. Zuccaro G. Management of the adult patient with acute lower gastrointestinal bleeding. American College of Gastroenterology. Practice Parameters Committee. Am J Gastroenterol 1998; 93: 1202-8.

19. Browder W, Cerise EJ, Litwin MS. Impact of emergency angiography in massive lower gastrointestinal bleeding. Ann Surg 1986; 204: 530-6.

20. DeBarros J, Rosas L, Cohen J et al. The changing paradigm for the treatment of colonic hemorrhage. Superselective angiographic embolization. Dis Colon Rectum 2002; 45: 802-8.

21. Khanna A, Ognibene SJ, Koniaris LG. Embolization as first-line therapy for diverticulosis-related massive lower gastrointestinal bleeding: evidence from a meta-analysis. J Gastrointest Surg 2005; 9: 343-52.

22. Hoedema RE, Luchtefeld MA. The management of lower gastrointestinal hemorrhage. Dis Colon Rectum 2005; 48: 2010-24.

23. Laine L, Smith R, Min K, Chen C, Dubois RW. Systematic review: the lower gastrointestinal adverse effects of non-steroidal anti-inflammatory drugs. Aliment Pharmacol Ther 2006; 24: 751-67. 
24. Parkes BM, Obeid FN, Sorensen VJ et al. The management of massive lower gastrointestinal bleeding. Am Surg 1993; 59: 676-8.

25. Jeyarajah S, Faiz O, Bottle A, et al. Diverticular disease hospital admissions are increasing, with poor outcomes in the elderly and emergency admissions. Aliment Pharmacol Ther 2009; 30: 1171-82.

26. Bender JS, Wiencek RG, Bouwman DL. Morbidity and mortality following total abdominal colectomy for massive lower gastrointestinal bleeding. Am Surg 1991; 57: 536-41. 27. Smoot RL, Gostout CJ, Rajan E et al. Is early colonoscopy after admission for acute diverticular bleeding needed? Am J Gastroenterol 2003; 98: 1996-9.

28. Breen E, Murray JJ. Pathophysiology and natural history of lower gastrointestinal bleeding. Semin Colon Rectal Surg 1997; 8: 128-38. 
1

2

3

4

5

6

7

8

9

10

11

12

13

14

15

16

17

18

19

20

21

22

23

24

25

26

27

28

29

30

31

32

33

34

35

36

37

38

39

40

41

42

43

44

45

46

47

48

49

50

51

52

53

54

55

56

57

58

59

60

Table 1: Biological data of patients admitted for colonic diverticular bleeding

\begin{tabular}{|l|c|}
\hline Biological variables & Mean (ranges) \\
\hline Admission haemoglobin level (g/L) & $115.18(42-164)$ \\
\hline Lowest haemoglobin level during hospital stay (g/L) & $93.42(42-147)$ \\
\hline Discharge haemoglobin level (g/L) & $105.98(82-147)$ \\
\hline Admission platelets level $\left(\mathrm{x} 10^{3} / \mathrm{mm}^{3}\right)$ & $246.82(102-837)$ \\
\hline Admission Prothrombin level $(\%)$ & $68.14(9-100)$ \\
\hline
\end{tabular}


Table 2: Comparison between rebleeding and non-rebleeding groups

\begin{tabular}{|l|c|c|c|}
\hline & Rebleeding group & Non-rebleeding group & $\mathrm{p}$ \\
\hline Number of patients & 17 & 113 & \\
\hline Sex ratio (males/females) & $8 / 9$ & $63 / 50$ & NS \\
\hline Mean age (range) & $74.1(48-88)$ & $75.3(51-97)$ & NS \\
\hline Patients with no medication (\%) & $3(17.6)$ & $15(13.2)$ & NS \\
\hline
\end{tabular}

Comment [UW14]: This table has been added to give answers to reviewers 2 and 3 . In the same time, the figure and tables 1 and 2 from the previous version have been deleted 